\title{
Design and Construction of Accelerator-Based Boron Neutron Capture Therapy (AB-BNCT) Facility with Multiple Treatment Rooms at Southern Tohoku BNCT Research Center
}

Takahiro Kato ${ }^{1,2}$, Katsumi Hirose ${ }^{1}$, Kazuhiro Arai ${ }^{1}$, Tomoaki Motoyanagi ${ }^{1}$, Takaomi Harada1, Akihiko Takeuchi ${ }^{1}$, Ryohei Kato ${ }^{1}$, Hiroki Tanaka ${ }^{3}$, Toshinori Mitsumoto ${ }^{4}$, Yoshihiro Takai ${ }^{1}$

'Southern Tohoku BNCT Research Center, Koriyama, Japan

${ }^{2}$ Fukushima Medical University, Fukushima, Japan

${ }^{3}$ Institute for Integrated Radiation and Nuclear Science, Kyoto University, Osaka, Japan

${ }^{4}$ Sumitomo Heavy Industires, Ltd., Tokyo, Japan

\section{Introduction and Purpose}

Boron neutron capture therapy (BNCT) is used to selectively destroy cancer cells injected with ${ }^{10} \mathrm{~B}$ compound, and currently, the research on accelerator-based BNCT (AB-BNCT) is being aggressively pursued all over the world. In contrast, thus far, not only $A B-B N C T$ facilities but also reactor-based BNCT facilities had only one treatment room, thus, limiting the number of patients who could benefit from BNCT. Therefore, it is necessary to design a facility with multiple treatment rooms to treat more patients, similar to highvolume centers for radiotherapy or particle beam therapy.

AB-BNCT facility was designed and constructed at Southern Tohoku BNCT Research Center (STBRC) to improve treatment outcome for intractable cancers such as malignant brain tumors and advanced head and neck cancers. In November 2015, STBRC was inaugurated as the first hospital-based BNCT facility in the world. In this study, we describe the design and construction of the AB-BNCT facility with multiple treatment rooms at STBRC.

\section{Materials and Methods}

\section{Design of beamline and layout of multiple treatment rooms}

The AB-BNCT system consists of a cyclotron accelerator, named HM-30 (Sumitomo Heavy Industries, Ltd.), that can produce a $1 \mathrm{~mA}$ or higher proton beam with an energy of $30 \mathrm{MeV}$, a beam transport system, a beryllium neutron production target, a beam shaping assembly (BSA), a collimator assembly, and patient setting-up system. We designed the upside-down, Y-shaped beamline configuration, in which the $\mathrm{HM}-30$ and two treatment rooms were to be located on the top and bottoms, respectively (Fig. 1).

We developed a remote patient carrying system (RPCS) to reduce hospital staff work time in the treatment room and to maintain a sufficient distance from the treatment room (Fig. 2). We setup a patient in the preparation room located in front of the treatment room and adopted the method of sending the patient to the treatment room remotely. Employing this method eliminates the need for medical stuffs to work in an activated treatment

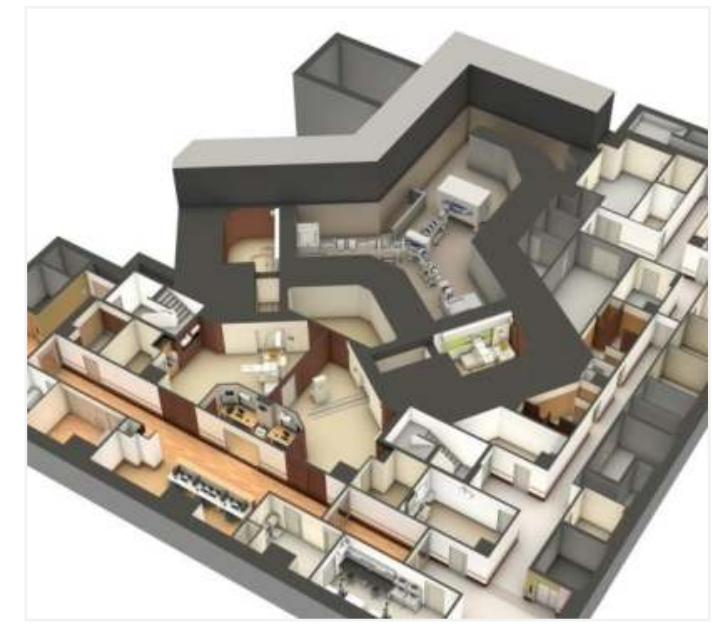

Fig. 1 An overview of the STBRC.

room just after irradiation and sufficient distance from the treatment room and can be expected to reduce cumulative exposure for medical stuffs.

- Evaluation of beam quality equivalency between two rooms

To detect thermal neutron flux depth profile, gold wires and cadmiumcovered gold wires were positioned at the central axis in water phantom (Fig. 3), to detect thermal neutron flux. After irradiation, the activities of the gold wires were measured by a high-purity germanium detector. Gamma-rays were also measured using thermoluminescent dosimeters (TLDs) on the central axis. The measured profiles were compared with the calculated profiles using the Monte Carlo simulation code MCNPX with cross-section data ENDF/B-VII. In addition, an out-of-field thermal neutron, fast neutron and gamma-ray dose profiles were measured in front of the collimator to evaluate effective radiation protection.

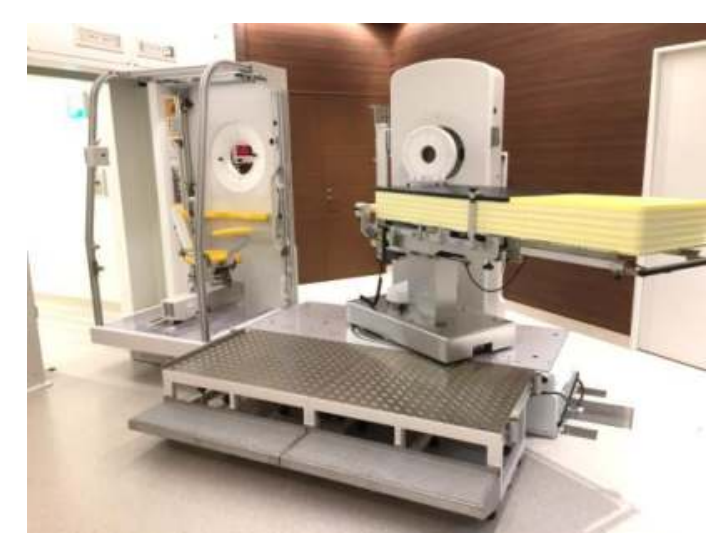

Fig. 2 An overview of the RPCS.

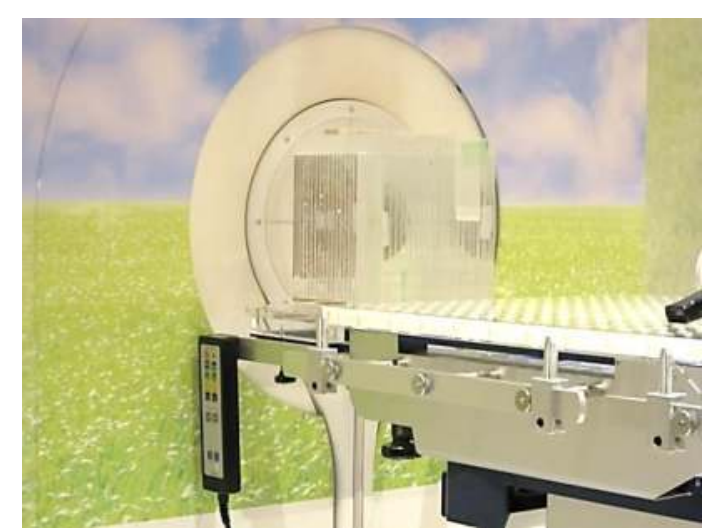

Fig. 3 An overview of the cubic water pantom.
Fig. 4 shows the measured thermal neutron depth profiles in a water phantom compared with the calculated results. It was confirmed that the measured profiles of both treatment rooms and calculated profile were within gold wire activation method errors of less than $8 \%$.

Fig. 5 shows the measured gamma-ray depth profiles in a water phantom compared with the calculated results. It was also confirmed that the measured profiles of both treatment rooms and calculated profile were within TLDs errors of less than $10 \%$. Since the results for other collimator sizes $(10,15,25 \mathrm{~cm}$ diameter) are similar tendency, beam quality equivalency between the two treatment rooms was confirmed.

The results of out-of-field thermal neutron and gamma-ray dose profiles in air in the lateral direction for the two treatment rooms are shown in Fig. 6 and 7 , respectively. It was confirmed that the results of the two treatment rooms were consistent within the range of the error of each measurement.

\section{Results}

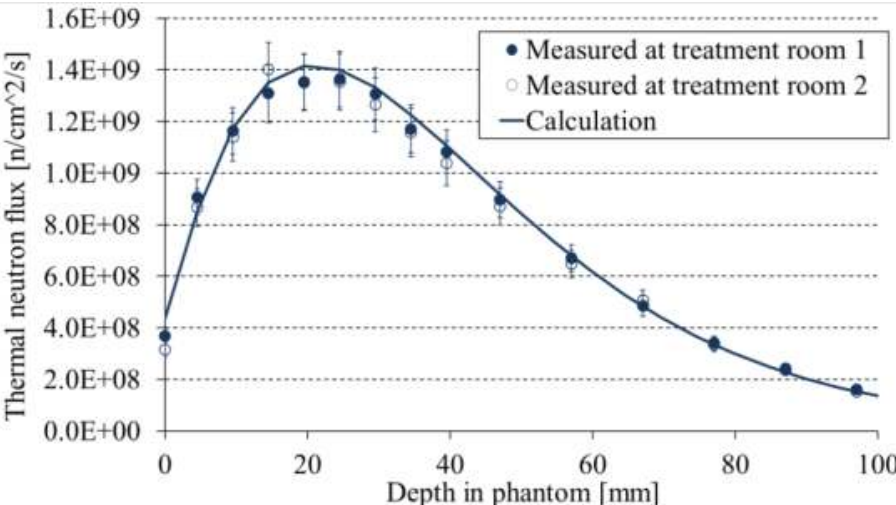

Fig. 4 Measured and calculated thermal neutron depth profiles ( $12 \mathrm{~cm} \varphi$ collimator)

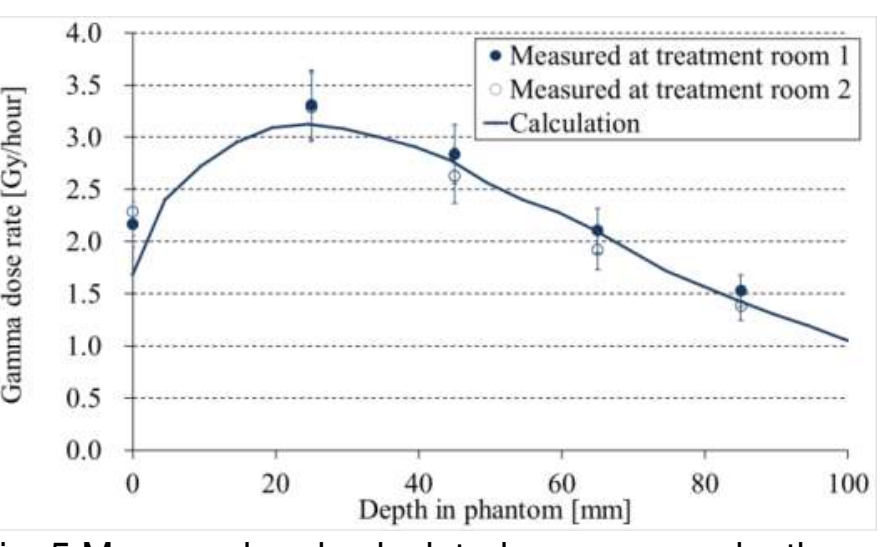

Fig. 5 Measured and calculated gamma-ray depth profiles ( $12 \mathrm{~cm} \varphi$ collimator).

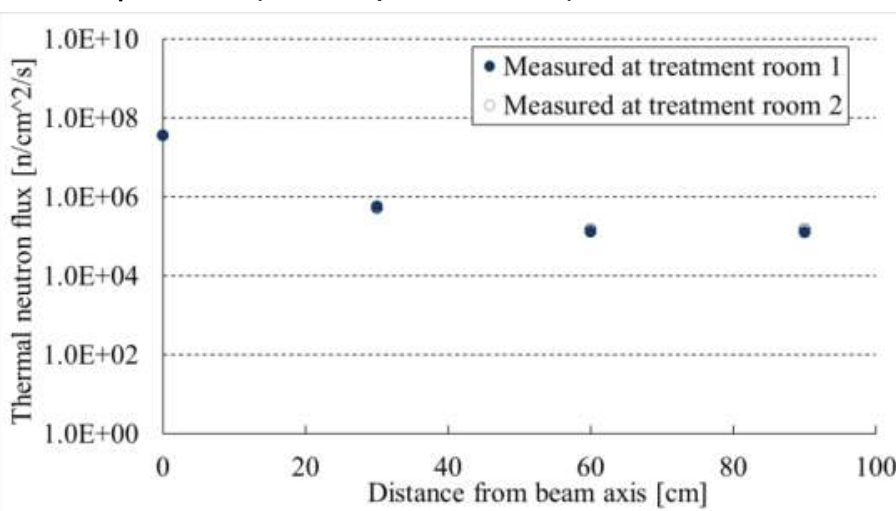

Fig. 6 Measured out-of- field thermal neutron profiles in air $(25 \mathrm{~cm} \varphi$ collimator)

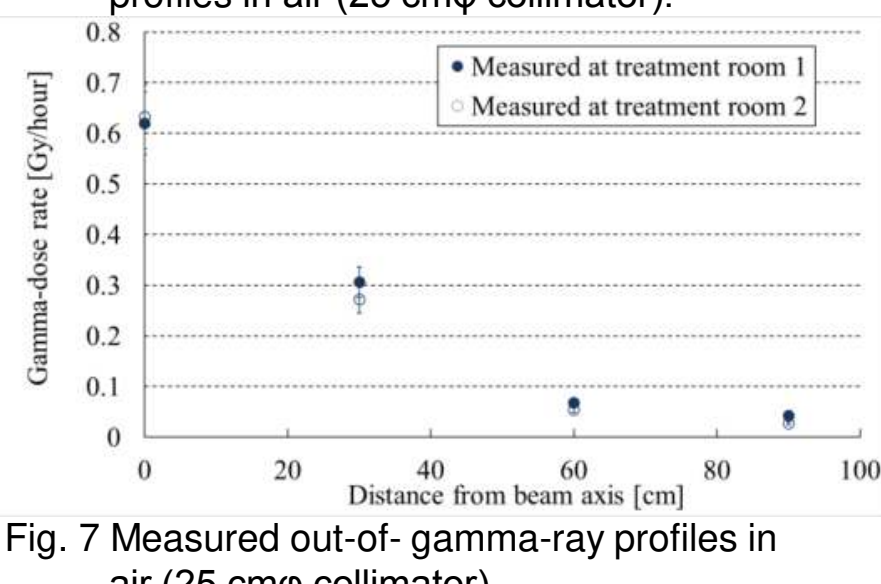
air $(25 \mathrm{~cm} \varphi$ collimator)

\section{Discussion}

We confirmed that beam quality equivalency between the two treatment rooms and sufficient epithermal neutron yield based on the ${ }^{9} \mathrm{Be}(\mathrm{p}, \mathrm{n})^{9} \mathrm{~B}$ reaction using optimum $\mathrm{BSA}$, which can be used in the clinical applications of BNCT. Although there was some disagreement in measured data between two treatment rooms, the trend seems to be similar. These differences are not considered to be a clinically problematic level, therefore, we decided to use identical beam data for both treatment rooms and registered it in the treatment planning system.

It was necessary to devise structures for reinforcing radiation protection and reducing radiation activity because reactions between $30 \mathrm{MeV}$ protons and the beryllium target emit high-energy neutrons. Our results indicate that the shielding of thermal neutron, fast neutron and gamma-ray is functioning effectively. We expect this reduction also lowers whole-body exposure in patients being treated with this system.

\section{Conclusion}

To increase radiation protection, to reduce additional exposure of hospital staff from radiation activities, and to realize workflow efficiency, we were able to establish BNCT as a part of general hospital without any apparent incongruity when compared with the environment of conventional radiotherapy. The AB-BNCT system described in this study was confirmed to satisfy specifications to be used for BNCT in a hospital. 